03

\title{
О физическом смысле дисперсионных параметров частотной зависимости диэлектрической проницаемости в модели Гавриляка-Негами
}

\author{
(С) А.С. Волков, Г.Д. Копосов, Р.О. Перфильев \\ Северный (Арктический) федеральный университет им. М.В. Ломоносова, \\ 163002 Архангельск, Россия \\ e-mail: a.s.volkov@narfu.ru
}

Поступила в редакцию 02.03.2018г.

В окончательной редакции 23.05.2018г.

\begin{abstract}
Работа посвящена анализу частотной дисперсии диэлектрической проницаемости на предмет установления физического смысла дисперсионных параметров $\alpha$ и $\beta$ в модели Гавриляка-Негами. Установлено, что параметр $\alpha$ связан с уширением спектра времен релаксации $\tau$. Представлены примеры с появлением дисперсии с параметром $\alpha$ около 0.4 и 0.7. Сделан вывод о том, что неупорядоченность порождает размытие времен релаксации. Для сохранения единого времени релаксации вводятся дисперсионные параметры $\alpha$ и $\beta$. Показана возможность анализа экспериментальных результатов на основе модели Дебая с целью получения распределения времени релаксации и определения по графику $\lg \left(\tau_{H N} / \tau\right)$ параметров $\alpha$ и $\beta$.
\end{abstract}

DOI: $10.21883 /$ OS.2018.09.46552.64-18

\section{Введение}

В физике диэлектриков сложилось противоречие между уровнем математического описания и физического содержания в описании частотной зависимости диэлектрической проницаемости. Существующие микроскопические теории релаксационной поляризации неизменно обосновывают существование дебаевской частотной дисперсии. Развитие экспериментов привело к тому, что экспериментальные факты не всегда описывались теорией Дебая [1]. С 40-х годов XX века стали последовательно вводиться модели Коул-Коула [2], КоулаДэвидсона [3], и Гавриляка-Негами [4]. Последняя модель является обобщением предыдущих и имеет вид

$$
\varepsilon=\varepsilon_{\infty}+\frac{\varepsilon_{s}-\varepsilon_{\infty}}{\left[1+\left(j \omega \tau_{0}\right)^{1-\alpha}\right]^{\beta}} .
$$

В этой модели действительная и мнимая компоненты комплексной диэлектрической проницаемости находятся следующим образом [4]:

$$
\begin{gathered}
\varepsilon^{\prime}=\varepsilon_{\infty}+\left(\varepsilon_{s}-\varepsilon_{\infty}\right) r^{-\beta / 2} \cos \beta \theta, \\
\varepsilon^{\prime \prime}=\left(\varepsilon_{s}-\varepsilon_{\infty}\right) r^{-\beta / 2} \sin \beta \theta
\end{gathered}
$$

где

$$
\begin{aligned}
r=[1 & \left.+(\omega \tau)^{1-\alpha} \sin \left(\frac{\alpha \pi}{2}\right)\right]^{2} \\
+ & {\left[\left(\omega \tau_{0}\right)^{1-\alpha} \cos \left(\frac{\alpha \pi}{2}\right)\right]^{2}, } \\
\theta=\operatorname{arctg} & {\left[\frac{\left(\omega \tau_{0}\right)^{1-\alpha} \cos \left(\frac{\alpha \pi}{2}\right)}{1+\left(\omega \tau_{0}\right)^{1-\alpha} \sin \left(\frac{\alpha \pi}{2}\right)}\right] . }
\end{aligned}
$$

Модель Гавриляка-Негами (1) переходит в другие вышеназванных модели частотной дисперсии:

- при $\alpha=0$ и $\beta=1$ - модель Дебая;

- при $1>\alpha>0$ и $\beta=1$ - модель Коул-Коула;

- при $\alpha=0$ и $1>\beta>0$ - модель Дэвидсона-Коула.

В предыдущей работе [5] авторы предложили модель анализа экспериментальных результатов по диэлектрической спектроскопии на предмет отождествления с моделью Гавриляка-Негами.

При этом микроскопической теории релаксационной поляризации, обосновывающей эту модель, не существует. Настоящая работа имеет своей целью обратить внимание на физическую сущность, которая заложена в дисперсионных параметрах $\alpha$ и $\beta$.

\section{Связь дисперсионных параметров $\alpha$ и $\beta$ с расширением частотной дисперсии}

Для установления связи воспользуемся методом числового моделирования. Влияние параметров $\alpha$ и $\beta$ на вид частотной дисперсии мнимой части $\varepsilon_{r e l}^{\prime \prime}$ комплексной диэлектрической проницаемости иллюстрирует рис. $1, a, b$.

Из рис. $1, a$ видно, что параметр $\alpha$ связан с расширением частотной дисперсии от $10^{2}$ (при $\alpha=0$ ) до $10^{7} \mathrm{~Hz}$ (при $\alpha=0.6)$. Параметр $\beta$ при уменьшении от 1 до 0.5 уширяет дисперсионную кривую в сторону больших частот от $10^{2}$ до $10^{4} \mathrm{~Hz}$.

Аналогичные выводы можно сделать из анализа частотной дисперсии действительной части $\varepsilon^{\prime}$.

Из сказанного следует вывод: введение дисперсионных параметров $\alpha$ и $\beta$ отражает уширение дисперсионных кривых по частоте. 


\section{Связь функции распределения времен релаксации с дисперсионными параметрами $\alpha$ и $\beta$}

Обобщенная формула частотной дисперсии диэлектрической проницаемости по отношению к уравнению (1) имеет вид [5]

$$
\varepsilon=\varepsilon_{\infty}+\left(\varepsilon_{s}-\varepsilon_{\infty}\right) \int_{0}^{\infty} \frac{g(\tau) d \tau}{1+j \omega \tau_{H N}}
$$

Функция распределения времен релаксации для модели частотной дисперсии Гавриляка-Негами [5]

$$
\begin{aligned}
& g(r)=\frac{1}{\pi} \\
& \times \frac{\left(\tau / \tau_{H N}\right)^{\beta(1-\alpha)} \sin (\beta \varphi)}{\left[\left(\tau / \tau_{H N}\right)^{2(1-\alpha)}+2\left(\tau / \tau_{H N}\right)^{1-\alpha} \cos (\pi[1-\alpha])+1\right]^{\beta / 2}},
\end{aligned}
$$

где

$$
\varphi=\operatorname{arctg}\left[\frac{\sin (\pi[1-\alpha])}{\left(\tau / \tau_{H N}\right)+\cos (\pi[1-\alpha])}\right] .
$$

В уравнениях (4) и (5) предполагается, что время релаксации $\tau_{0}$ является единым и в модели ГаврилякаНегами как $\tau_{H N}$.

Вид функции $g(\tau)$ при различных значениях параметра $\alpha$ представлен на рис. 2 [5]. Из рис. 2 видно, что увеличение параметра $\alpha$ снижает высоту максимума функции $g(\tau)$, смещает положение максимума по шкале $\left(\tau / \tau_{H N}\right)$ в область меньших значений и уширяет спектр времен релаксации.

С позиции спектроскопического подхода необходимо проанализировать связь ширины спектральной функции, определяющей уширение $\Delta\left(\tau / \tau_{H N}\right)$, на уровне $g_{\max }(\tau) / 2$ с дисперсионными параметрами. На рис. 3 представлена зависимость $\lg \Delta\left(\tau / \tau_{H N}\right)$ от $\alpha$ в областях высоких и низких частот, а так же их сумма.

Из проведенного анализа можно высказать предположение о том, что дисперсионные параметры $\alpha$ и $\beta$ отражают уширение распределения по временам релаксации. Уширение функции распределения времен релаксации согласно соотношению $\tau=\left(1 / 2 v_{r e l}\right) \exp (\Delta E / k T)$ может быть связано с двумя причинами: первая - размытие собственных частот релаксаторов $v_{\text {rel }}$ и вторая - размытие энергии активации релаксаторов.

\section{Экспериментальные результаты}

В поисках экспериментальных фактов изменения параметров $\alpha$ мы обратились ко льду. Известно [6,7], что в монокристаллическом льду дисперсия диэлектрической проницаемости является дебаевской. При переходе к мерзлым дисперсным средам, когда ледяные пленки покрывают гранулы вещества, на границе гранула-лед следует ожидать изменения частоты осцилляторов и,
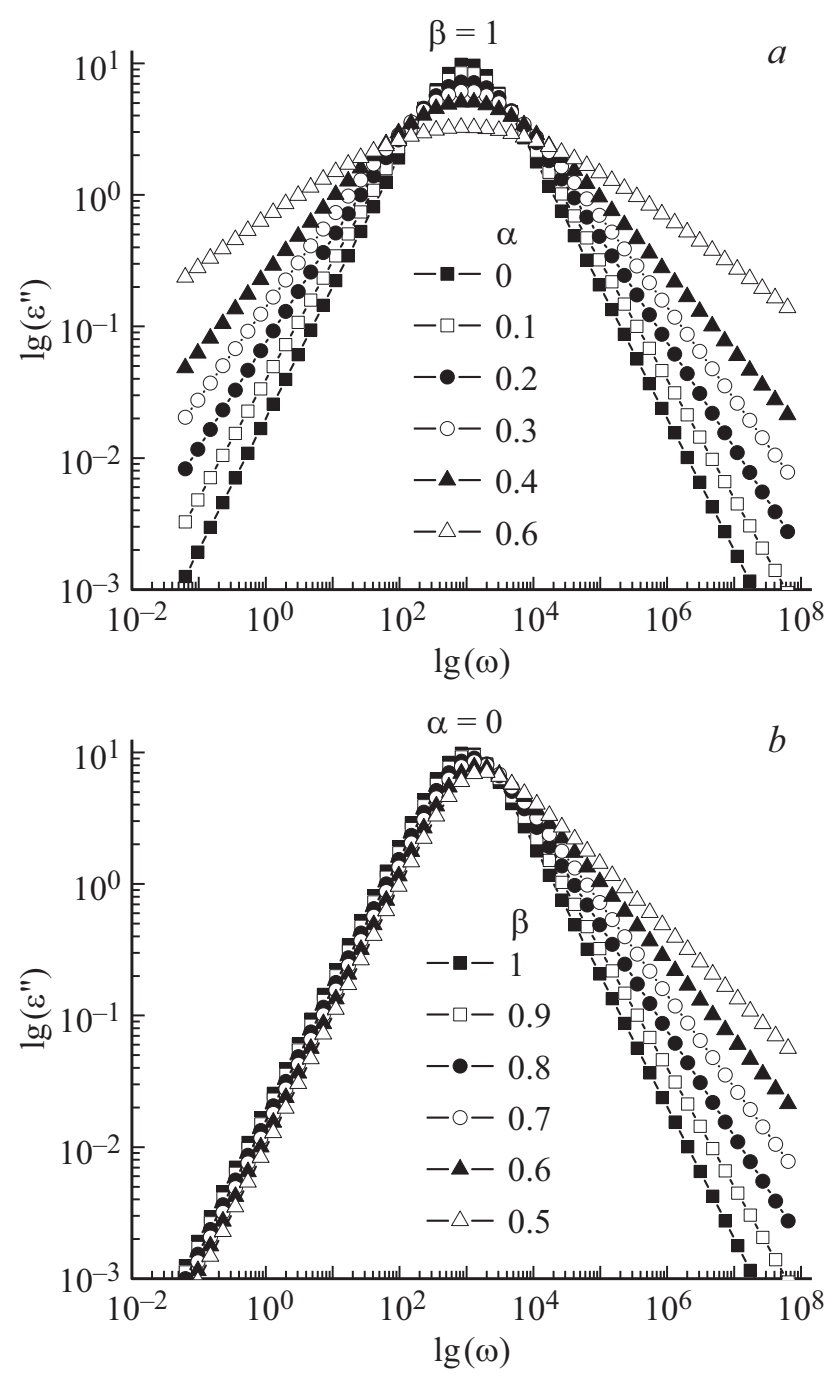

Рис. 1. Влияние параметров $\alpha$ при $\beta=1$ (a) и $\beta$ при $\alpha=0(b)$ на частотную дисперсию мнимой части комплексной диэлектрической проницаемости $\left(\varepsilon_{s}-\varepsilon_{\infty}=20 ; \tau_{H N}=10^{-3} \mathrm{~s}\right)$.

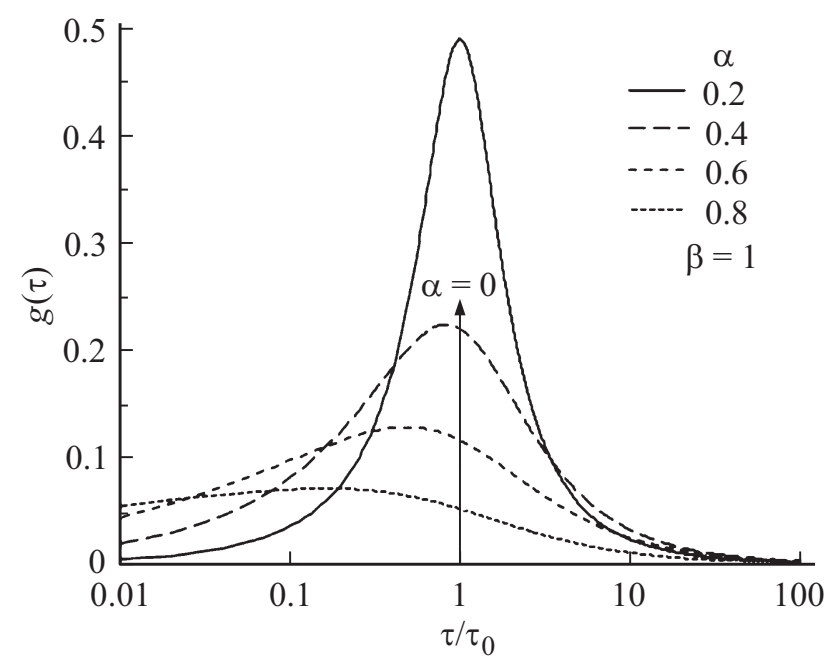

Рис. 2. Вид функции распределения $g(\tau)=f\left(\tau / \tau_{H N}\right)$ при $\beta=1$ и различных значениях параметра $\alpha[5]$. 


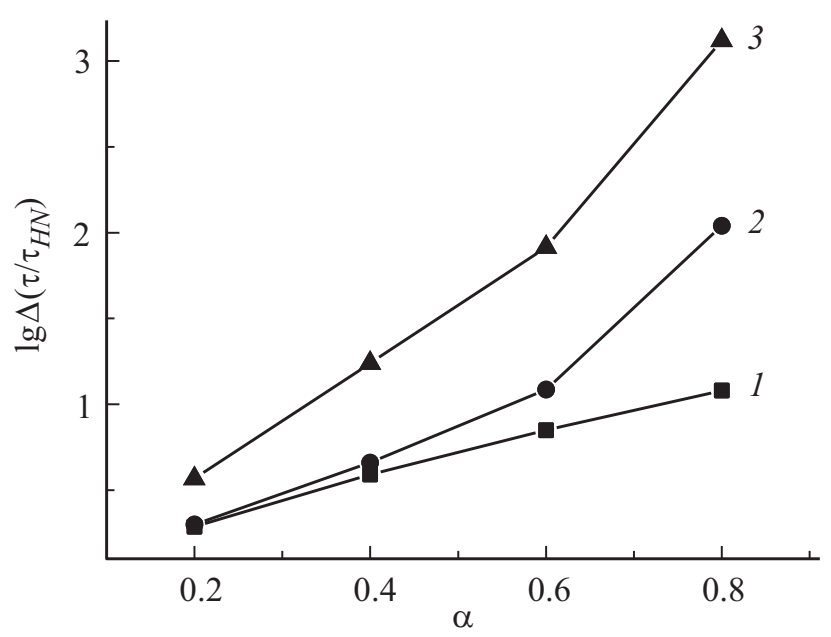

Рис. 3. Зависимость ширины функции распределения по времени релаксации от параметра $\alpha$ в областях высоких (1) и низких (2) частот, а также суммарной (3).

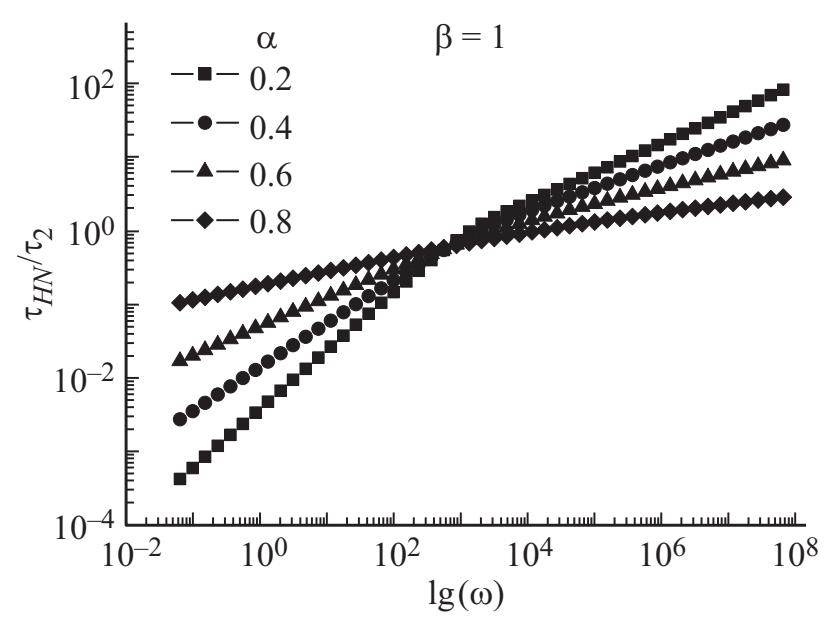

Рис. 4. Зависимость $\tau_{H N} / \tau=f(\omega)$ при $\beta=1$ и различных значениях $\alpha$.

следовательно, уширение $v_{\text {rel }}$. Кроме этого, возникает и уширение в энергии активации по причине различия их на границе гранул льда и в объеме и при поликристалличности образцов. Исследование температурночастотных зависимостей действительной $\varepsilon^{\prime}$ и мнимой $\varepsilon^{\prime \prime}$ частей диэлектрической проницаемости мерзлой дисперсной среды на основе порошка кварца проводилась на диэлектрическом спектрометре ВДС Novocontrol Concept 80 в диапазоне температур $T=133-273 \mathrm{~K}$ и диапазоне частот от $10^{-2}$ до $10^{6} \mathrm{~Hz}$. Обработка частотных зависимостей $\varepsilon_{r e l}^{\prime \prime}=\varepsilon^{\prime \prime}-\left(\sigma_{0} / \varepsilon_{0} \omega\right)$ и $\varepsilon^{\prime}$ по программе, представленной в работе [8], позволила определить дисперсионные параметры $\alpha$ и $\beta$. В результате исследований в диапазоне влажностей от 3 до $13 \%$ получены значения $\alpha=0.42 \pm 0.01$ и $\beta=0.98 \pm 0.01$.

В работе [9] представлены исследования пленок из наночастиц $\mathrm{Si}$, результаты свидетельствуют о выполнимости модели Коул-Коула при $\alpha \sim 0.7$. При этом пред- ставлена модель объяснения частотной зависимости проводимости $\sigma(v)=\sigma_{S}+A v^{S}$ при $S=0.74$. Число же $S$ оказалось связанным с фрактальной размерностью $d_{f}$ : $S=d_{f} / 2$. Это подтверждает утверждение о том, что параметр $\alpha$ связан с неупорядоченностью структуры вещества.

\section{Частотная зависимость времени релаксации по модели Дебая}

Соотношение Коула-Коула, как известно, справедливо в том случае, когда в веществе присутствуют одновременно несколько типов релаксаторов, каждый со своим временем релаксации. Этот факт может быть выявлен методом численного моделирования в дебаевском приближении. Будем полагать, что $\omega \tau=1$, т. е. на частоте $\omega$ реагируют релаксаторы с $\tau=1 / \omega$, тогда в дебаевском приближении

$$
\varepsilon^{\prime}=\varepsilon_{\infty}+\frac{\varepsilon_{s}-\varepsilon_{\infty}}{1+\left(\omega \tau_{H N}\right)^{2}}=\varepsilon_{\infty}+\frac{\varepsilon_{s}-\varepsilon_{\infty}}{1+\left(\tau_{H N} / \tau\right)_{\omega}^{2}}
$$

Из этого следует

$$
\frac{\tau_{H N}}{\tau}=\sqrt{\frac{\varepsilon_{s}-\varepsilon^{\prime}(\omega)}{\varepsilon^{\prime}(\omega)-\varepsilon_{\infty}}}=\sqrt{\frac{\varepsilon_{s}-\varepsilon_{\infty}}{\varepsilon^{\prime}-\varepsilon_{\infty}}-1}=f(\omega) .
$$

Выясним зависимость $\tau_{H N} / \tau=f(\omega)$ от $\alpha$ и $\beta$. Для выяснения этой зависимости проведем модельный расчет при $\tau_{H N}=10^{-3} \mathrm{~s}, \varepsilon_{\infty}=3, \varepsilon_{S}=13$ по модели ГаврилякаНегами

$$
\frac{\tau_{H N}}{\tau}=\sqrt{\frac{r^{\beta / 2}}{\cos (\beta \theta)}-1} \text { или } \frac{\tau}{\tau_{H N}}=\left(\frac{r^{\beta / 2}}{\cos (\beta \theta)}-1\right)^{-1 / 2},
$$

где $r$ и $\theta$ определяются по уравнениям (4) и (5).

Из рис. 4 следует, что $\tau_{H N} / \tau$ имеет значение, близкое к 1 при $\omega=1 / \tau$ и в области $\tau_{H N} / \tau>1$ (малые частоты) $\left(d \lg \left(\tau_{H N} / \tau\right) / d \lg \omega\right)=1-\alpha$. В области $\tau_{H N} / \tau \sim 1$ зависимость $\lg \left(\tau_{H N} / \tau\right)$ от $\lg \omega$ претерпевает излом и $\left(d \lg \left(\tau_{H N} / \tau\right) / d \lg \omega\right)$ становится близкой к $(1-\alpha)(\beta / 2)$.

Заметим, что поведение графиков может быть использовано для нахождения числовых значений дисперсионных параметров при введении в (10) экспериментальных значений $\varepsilon^{\prime}, \varepsilon_{S}$ и $\varepsilon_{\infty}$.

Величина $\tau$, входящая в уравнение, является временем релаксации, усредняемым по ансамблю группы релаксаторов, представляемых в исследуемом материале. Ранее в работе [10] эти результаты были представлены нами в виде частотной зависимости времени релаксации, которая получалась расчетом $\tau$ в квазидебаевском приближении

$$
\tau=\frac{1}{\omega} \sqrt{\frac{\varepsilon_{S}-\varepsilon^{\prime}}{\varepsilon^{\prime}-\varepsilon_{\infty}}}
$$

или

$$
\tau_{H N}=\frac{1}{\omega} \frac{\varepsilon_{r e l}^{\prime \prime}}{\varepsilon^{\prime}-\varepsilon_{\infty}} .
$$


Таким образом, во-первых, в дебаевском приближении переход к модели Гавриляка-Негами связан с появлением зависимости времени релаксации $\tau$ от частоты $\omega$. Во-вторых, методом математического моделирования установлено, что $\lg \left(\tau_{H N} / \tau\right)$ является линейной функцией от $\lg (\omega)$, зависящей от параметров $\alpha$ и $\beta$. В-третьих, анализ зависимости $\lg \left(\tau_{H N} / \tau\right)=f(\lg (\omega))$ позволяет найти параметры $\alpha$ и $\beta$ по тангенсу угла наклона. Точка перегиба на рис. 4 по оси $\lg (\omega)$ позволяет определить частоту $\omega^{\prime}$, соответствующую перегибу, и время релаксации $\tau_{H N}=\left(\omega^{\prime}\right)^{-1}$.

\section{Заключение}

Резюмируя, можно утверждать, что введение дисперсионных параметров $\alpha$ и $\beta$ в распределениях диэлектрической проницаемости связано с ростом неупорядоченности в исследуемых материалах, что выражается, в частности, в зависимости $\alpha$ от фрактальной размерности, и не случайно, что распределение ГаврилякаНегами было введено для описания полимерных структур.

Кроме этого, можно сделать вывод, что неупорядоченность порождает размытие частот колебаний релаксаторов и в соответствии с уравнением $\tau=\left(1 / 2 v_{r e l}\right) \exp (\Delta E / k T)$ проявляется в размытии времени релаксации.

Введение дисперсионных параметров $\alpha$ и $\beta$ осуществляется во имя спасения единого времени релаксации. Обработка экспериментальных результатов возможна по дебаевской модели, но с добавлением исследования зависимости дебаевского времени релаксации от частоты электрического поля.

\section{Список литературы}

[1] Debye P. Polar Molecules: Wisconsin Lectures. NY.: Chemical Catalog Co., 1929; Дебай П. Полярные молекулы. М.-Л.: ГНТИ, 1931. $241 \mathrm{c.}$

[2] Cole K.S., Cole R.H. // J. Phys. Chem. 1941. V. 9. P. 341.

[3] Devidson D.W., Cole R.H. // J. Chem. Physic. 1951. V. 19. P. 1484.

[4] Havriliak S., Negami S. // J. Polym. Sci. C. 1966. V. 14. P. 99.

[5] Ramos A., Pezzin S.H., Farias H.D., Becker D., Bello R.H. // Physica B. 2016. V. 499. P. 57. doi 10.1016/j.physb.2016.07.016

[6] Тонконогов М.П. // УФН. 1998. Т. 168. С. 29.

[7] Petrenko V.F., Whitworsh R.W. Physics of Ice. NY:: Oxford University Press, 2006. 373 p.

[8] Волков А.С., Копосов Г.Д., Перфильев Р.О., Тягунин А.B. // Опт. и спектр. 2018. Т. 124. № 2. С. 206. doi 10.21883/OS.2018.02.45525.200-17

[9] Ищенко А.А., Фетисов Г.В., Аслант А.А. Нанокремний: свойства, получение, применение, методы исследования и контроль. М.: Физматлит, 2011. 647 с.

[10] Volkov A.S., Koposov G.D., Tyagunin A.V. // ARPN Journal of Engineering and Applied Sciences. 2015. V. 10. P. 7929. 